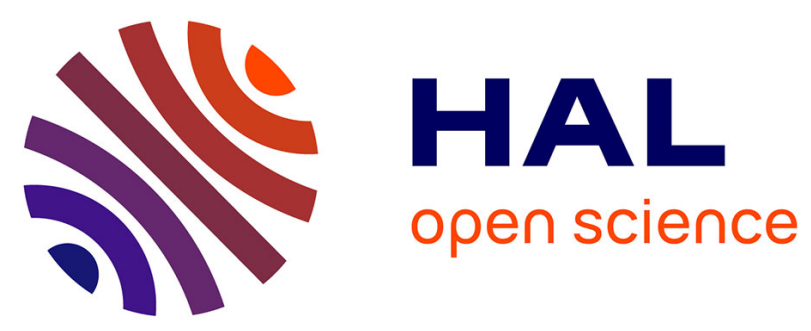

\title{
Double cross polarization for the indirect detection of nitrogen-14 nuclei in magic angle spinning NMR spectroscopy
}

Diego Carnevale, Xiao Ji, Geoffrey Bodenhausen

\section{- To cite this version:}

Diego Carnevale, Xiao Ji, Geoffrey Bodenhausen. Double cross polarization for the indirect detection of nitrogen-14 nuclei in magic angle spinning NMR spectroscopy. Journal of Chemical Physics, 2017, 147 (18), pp.184201. 10.1063/1.5000689 . hal-01637364

\section{HAL Id: hal-01637364 https: / hal.sorbonne-universite.fr/hal-01637364}

Submitted on 20 Nov 2017

HAL is a multi-disciplinary open access archive for the deposit and dissemination of scientific research documents, whether they are published or not. The documents may come from teaching and research institutions in France or abroad, or from public or private research centers.
L'archive ouverte pluridisciplinaire HAL, est destinée au dépôt et à la diffusion de documents scientifiques de niveau recherche, publiés ou non, émanant des établissements d'enseignement et de recherche français ou étrangers, des laboratoires publics ou privés. 


\section{Double cross polarization for the indirect detection of nitrogen-14 nuclei in magic angle spinning NMR spectroscopy}

Diego Carnevale, Xiao Ji, and Geoffrey Bodenhausen

Citation: The Journal of Chemical Physics 147, 184201 (2017);

View online: https://doi.org/10.1063/1.5000689

View Table of Contents: http://aip.scitation.org/toc/jcp/147/18

Published by the American Institute of Physics

\section{Articles you may be interested in}

Parameter independent low-power heteronuclear decoupling for fast magic-angle spinning solid-state NMR

The Journal of Chemical Physics 146, 084202 (2017); 10.1063/1.4976997

Off-resonance NOVEL

The Journal of Chemical Physics 147, 164201 (2017); 10.1063/1.5000528

Low-power broadband solid-state MAS NMR of ${ }^{14} \mathrm{~N}$

The Journal of Chemical Physics 146, 194202 (2017); 10.1063/1.4983220

Sine-squared shifted pulses for recoupling interactions in solid-state NMR

The Journal of Chemical Physics 146, 244201 (2017); 10.1063/1.4986791

Visualising crystal packing interactions in solid-state NMR: Concepts and applications

The Journal of Chemical Physics 147, 144203 (2017); 10.1063/1.4996750

Ramped-amplitude NOVEL

The Journal of Chemical Physics 146, 154204 (2017); 10.1063/1.4980155

\section{AIP| The Joumal of Chemical Physics}




\title{
Double cross polarization for the indirect detection of nitrogen-14 nuclei in magic angle spinning NMR spectroscopy
}

\author{
Diego Carnevale, ${ }^{1,2, a)}$ Xiao Ji, ${ }^{1,2}$ and Geoffrey Bodenhausen ${ }^{1,2}$ \\ ${ }^{1}$ Departement de Chimie, Ecole Normale Superieure, PSL Research University, UPMC Université Paris 06, \\ CNRS, Laboratoire des Biomolecules (LBM), 24 rue Lhomond, 75005 Paris, France \\ ${ }^{2}$ Sorbonne Universites, UPMC Université Paris 06, Ecole Normale Superieure, CNRS, Laboratoire des \\ Biomolecules (LBM), Paris, France
}

(Received 17 August 2017; accepted 16 October 2017; published online 9 November 2017)

\begin{abstract}
Nitrogen-14 NMR spectra at fast magic-angle spinning rates can be acquired indirectly by means of two-dimensional techniques based on double cross polarization transfer ${ }^{1} \mathrm{H} \rightarrow{ }^{14} \mathrm{~N} \rightarrow{ }^{1} \mathrm{H}$. Experimental evidence is given for polycrystalline samples of glycine, L-histidine, and the dipeptide Ala-Gly. Either one-bond or long-range correlations can be favored by choosing the length of the cross polarization contact pulses. Longer contact pulses allow the detection of unprotonated nitrogen sites. In contrast to earlier methods that exploited second-order quadrupolar/dipolar cross-terms, cross polarization operates in the manner of the method of Hartmann and Hahn, even for ${ }^{14} \mathrm{~N}$ quadrupolar couplings up to $4 \mathrm{MHz}$. Simulations explain why amorphous samples tend to give rise to featureless spectra because the ${ }^{14} \mathrm{~N}$ quadrupolar interactions may vary dramatically with the lattice environment. The experiments are straightforward to set up and are shown to be effective for different nitrogen environments and robust with respect to the $r f$-field strengths and to the ${ }^{14} \mathrm{~N}$ carrier frequency during cross polarization. The efficiency of indirect detection of ${ }^{14} \mathrm{~N}$ nuclei by double cross polarization is shown to be similar to that of isotopically enriched ${ }^{13} \mathrm{C}$ nuclei. Published by AIP Publishing. https://doi.org/10.1063/1.5000689
\end{abstract}

\section{INTRODUCTION}

Nitrogen is a fundamental and ubiquitous element that plays a key role in a variety of fields such as biology, organic chemistry, and materials science. Appearing both in amino acids and nucleotides-the building blocks of proteins and nucleic acids-nitrogen lies at the heart of the "structural patterns" upon which life is based on Earth. The possibility to acquire nitrogen NMR spectra is therefore essential. Unfortunately, its spin- $1 / 2$ isotope ${ }^{15} \mathrm{~N}$ has a very low natural abundance of ca. $0.37 \%$. Most NMR studies relying on ${ }^{15} \mathrm{~N}$, such as the popular investigations of the structure and dynamics of proteins, must resort to costly isotopic enrichment. In contrast, the highly abundant ${ }^{14} \mathrm{~N}$ isotope (ca. 99.63\%) has a quadrupolar nucleus with $I=1$. This feature results in (i) solution-state signals that are usually too broad to be observed, except for highly symmetrical ammonium ions and (ii) solidstate signals that suffer from inhomogeneous broadening up to several $\mathrm{MHz}$ if the environments have a low symmetry. In contrast to half-integer quadrupolar spins, nitrogen-14 does not have any central transition $m_{I}=+1 / 2 \leftrightarrow m_{I}=-1 / 2$, which is usually much easier to observe since it is only affected by small second-order interactions that typically lie in the $\mathrm{kHz}$ range. ${ }^{1}$ As a result, the NMR observation of ${ }^{14} \mathrm{~N}$ is very challenging, even in solid samples that are spinning about the magic angle.

a)Author to whom correspondence should be addressed: diego.carnevale@ ens.fr
A commonly employed technique for the direct observation of less abundant nuclei such as ${ }^{13} \mathrm{C}$ and ${ }^{15} \mathrm{~N}$ is cross polarization $(\mathrm{CP}){ }^{2,3}$ Typically CP exploits the transfer of magnetization from abundant high- $\gamma$ nuclei with $I=1 / 2$ to less abundant low- $\gamma$ nuclei of interest with $S=1 / 2$. The CP process yields a gain in sensitivity that approaches the ratio $\gamma_{\mathrm{I}} / \gamma_{\mathrm{S}}$. This transfer is mediated by dipolar couplings between the two nuclei. In order to achieve this transfer, the Hartmann-Hahn matching condition has to be fulfilled, ${ }^{2}$ with Rabi nutation frequencies $\omega_{\mathrm{I}}=\omega_{\mathrm{S}}$ or $\omega_{\mathrm{I}}=\omega_{\mathrm{S}} \pm n \omega_{\mathrm{R}}$ for static or spinning samples, respectively, and $n=1$ or 2 . For quadrupolar nuclei with $S>1 / 2, \mathrm{CP}$ is usually less effective. The sensitivity enhancement is more difficult to achieve and the matching conditions are more complex. The introduction of multiplequantum (MQ) ${ }^{4}$ and satellite-transition (ST) ${ }^{5}$ methods has led to increased interest in solid-state NMR of half-integer spins. As a consequence, interest in $\mathrm{CP}$ involving quadrupolar nuclei has been renewed. ${ }^{6-10}$ Nonetheless, mostly half-integer quadrupolar spins with $S=3 / 2,5 / 2$, or 7/2—all of which have a favorable central transition-have been investigated in this context, with only very few studies concerning nuclei with $S=1 .^{11-13}$

Current methods for solid-state ${ }^{14} \mathrm{~N}$ NMR spectroscopy rely on (i) piecewise acquisition ${ }^{14}$ or frequency-swept excitation $^{15-17}$ in static samples, (ii) magic angle spinning (MAS) spectra utilizing small flip angle pulses so as to achieve broad excitation profiles, ${ }^{18,19}$ (iii) overtone irradiation, ${ }^{20-28}$ or (iv) heteronuclear multiple-quantum correlation (HMQC) experiments for the indirect detection of ${ }^{14} \mathrm{~N}$ spectra via "spy" nuclei such as ${ }^{1} \mathrm{H}$ or ${ }^{13} \mathrm{C} .{ }^{29-43}$ The HMQC approach offers advantages 
in terms of sensitivity as magnetization on the target $\operatorname{spin}{ }^{14} \mathrm{~N}$ is created initially by transfer from ${ }^{1} \mathrm{H}$ and ${ }^{13} \mathrm{C}$ nuclei. Detailed reviews are available of both direct ${ }^{44}$ and indirect ${ }^{45}$ methods for the acquisition of nitrogen-14 NMR spectra.

In this letter, we investigate the use of double cross polarization (DCP) ${ }^{1} \mathrm{H} \rightarrow{ }^{14} \mathrm{~N} \rightarrow{ }^{1} \mathrm{H}$ for the indirect detection of nitrogen-14 spectra in powdered samples under MAS conditions. In contrast with early studies that utilized cross polarization for the indirect detection of double-quantum (DQ) nitrogen- 14 coherences in static single crystals, ${ }^{46,47}$ we shall focus on single-quantum (SQ) nitrogen-14 coherences in polycrystalline powders that are spinning rapidly about the magic angle.

\section{RESULTS AND DISCUSSION}

Figure 1 depicts the pulse sequence proposed in this study. In contrast to HMQC-type experiments, where either SQ or DQ ${ }^{14} \mathrm{~N}$ coherences are created via ${ }^{14} \mathrm{~N} r f$ pulses acting on anti-phase heteronuclear single-quantum spin states such as $H_{y} N_{z}$ and $H_{x} N_{z}{ }^{2}$ that result from an initial free evolution of $H_{x}$ coherence under scalar interactions or second-order cross terms, a simple cross-polarization step in the manner of Hartmann and Hahn will be used in this study to convert in-phase $H_{y}$ coherence directly into in-phase transverse $N_{x}$ magnetization. In terms of irreducible tensor operators $T_{l m}$ of rank $l$ and coherence order $m$, the HMQC approach that we and others have advocated for a spin pair with $I=1 / 2$ and $S=1$ may be depicted by $T^{I}{ }_{1 \pm 1} \rightarrow T^{I}{ }_{1 \pm 1} T^{S}{ }_{10}+T^{I}{ }_{1 \pm 1} T^{S}{ }_{20}$ followed by $r f$ irradiation applied to the ${ }^{14} \mathrm{~N}$ nuclei to create anti-phase SQ terms $T^{I}{ }_{1 \pm 1} T^{S}{ }_{1 \pm 1}+T^{I}{ }_{1 \pm 1} T^{S}{ }_{2 \pm 1}$ as well as anti-phase DQ terms $T^{I}{ }_{1 \pm 1} T^{S}{ }_{2 \pm 2}$. Such heteronuclear multiple quantum coherences tend to have short lifetimes since the $T^{I}{ }_{1 \pm 1}$ tensor components decay rapidly due to strong protonproton interactions. By contrast, the much simpler CP process can be described by $T^{I}{ }_{1 \pm 1} \rightarrow T^{S}{ }_{1 \pm 1}$, leading to the creation of pure in-phase ${ }^{14} \mathrm{~N}$ single-quantum coherence that may have a longer lifetime and hence give rise to narrower signals. This $T^{S}{ }_{1 \pm 1}$ state is then allowed to evolve under chemical shifts and second-order quadrupolar interactions during an evolution interval $t_{1}$ that must be stepped in rotor-synchronized time increments $\Delta t_{1}=n \tau_{\mathrm{R}}=n / v_{\mathrm{R}}(n=1,2,3 \ldots)$ so as to refocus the effects of first-order quadrupolar interactions that cancel at the top of rotational echoes, provided the magic angle is set accurately. An identical CP step is then used to transfer the ${ }^{14} \mathrm{~N}$ single-quantum $T^{S}{ }_{1 \pm 1}$ coherence back to the proton coherence $T^{I}{ }_{1 \pm 1}$, the signals of which are finally acquired. Importantly, since we are dealing with single-spin states $T^{S}{ }_{1 \pm 1}$ rather than product states such as $T^{I}{ }_{1 \pm 1} T^{S}{ }_{1 \pm 1}$ or $T^{I}{ }_{1 \pm 1} T^{S}{ }_{2 \pm 1}$, our double $\mathrm{CP}$ experiments do not need any $\pi$ pulse applied to the protons in the middle of the $t_{1}$ evolution interval in order to refocus modulations due to the offsets of the protons. Such refocusing pulses obviously fail to cancel the effects of proton-proton dipole-dipole interactions, which therefore contribute to the decay of $T^{I}{ }_{1 \pm 1} T^{S}{ }_{1 \pm 1}$ and $T^{I}{ }_{1 \pm 1} T^{S}{ }_{2 \pm 1}$ terms and hence to the linewidths in the $\omega_{1}$ dimension of HMQC-type experiments. These homogeneous losses-leading to linebroadenings-may obscure the inhomogeneous features due to second-order quadrupolar couplings and hinder the extraction of the quadrupolar coupling constant $C_{Q}$ and asymmetry parameter $\eta_{Q}$.

Figure 2 shows experimental 2D spectra acquired with our novel DCP approach for a polycrystalline powder of Lhistidine. The spectrum in Fig. 2(a) was acquired with a contact time $\tau_{C P}=240 \mu \mathrm{s}=15 \tau_{R}$ (e.g., $n=15$ ), a duration that favors one-bond transfers in this sample. Strong one-bond correlations arising from the $\mathrm{NH}_{3}{ }^{+}$and $\mathrm{N}^{\varepsilon 2} \mathrm{H}$ sites are clearly identified. Weaker correlations between the $\mathrm{NH}_{3}{ }^{+}$nitrogen-14 and more remote protons may also be distinguished. Figure 2(b) shows an analogous spectrum acquired with a longer contact time $\tau_{C P}=6.4 \mathrm{~ms}=400 \tau_{R}$ (e.g., $\left.n=400\right)$, a duration that allows polarization to be transferred between nitrogen-14 sites and both close and remote protons, so that all ${ }^{14} \mathrm{~N}$ sites appear to be correlated with all protons. Most importantly, the unprotonated $\mathrm{N}^{\delta 1}$ site is clearly identified in the latter spectrum. To the best of our knowledge, this nitrogen-14 site has never been observed before by indirect detection. The position of the carrier frequency $v_{r f}\left({ }^{14} \mathrm{~N}\right)$ in the spectrum of Fig. 2(b) leads to two horizontal ridges (corresponding to the $\mathrm{NH}_{3}{ }^{+}$and $\mathrm{N}^{\varepsilon 2} \mathrm{H}$ protons) that appear near the edge of the spectral width in the vertical $\omega_{1}$ domain. This finding suggests that the experiment is remarkably robust with respect to the position of the carrier frequency $v_{r f}\left({ }^{14} \mathrm{~N}\right)$. The ${ }^{14} \mathrm{~N}$ line shapes obtained in the $\omega_{1}$ projections of these two ridges reveal how second-order features and intensities may vary critically depending on the length of the CP contact time and the carrier position. For a given set of experimental parameters, the efficiency of the $\mathrm{CP}$ process may therefore not be uniform for all crystallite orientations, thus resulting in a partial excitation of the line shapes. The quadrupolar coupling constant $C_{Q}$ may therefore be underestimated, and the asymmetry parameter $\eta_{Q}$ derived from fitting the line shape may not be quite accurate.

Figure 3(a) shows the DCP spectrum of a polycrystalline powder of the dipeptide Ala-Gly acquired with a contact time

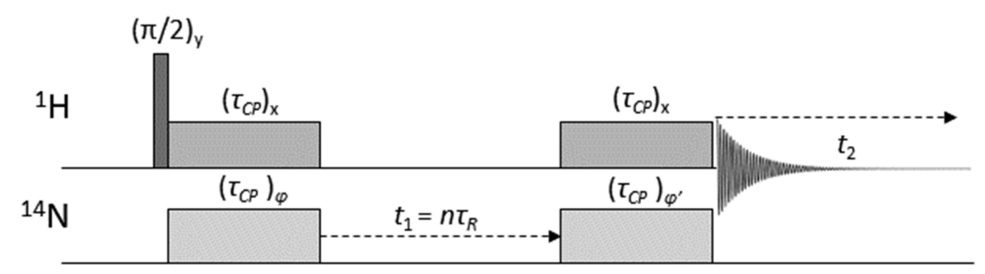

FIG. 1. Pulse sequence for double cross polarization (DCP) for the indirect detection of ${ }^{14} \mathrm{~N}$ signals with corresponding coherence transfer pathway. Phase cycling is given in the supplementary material. 


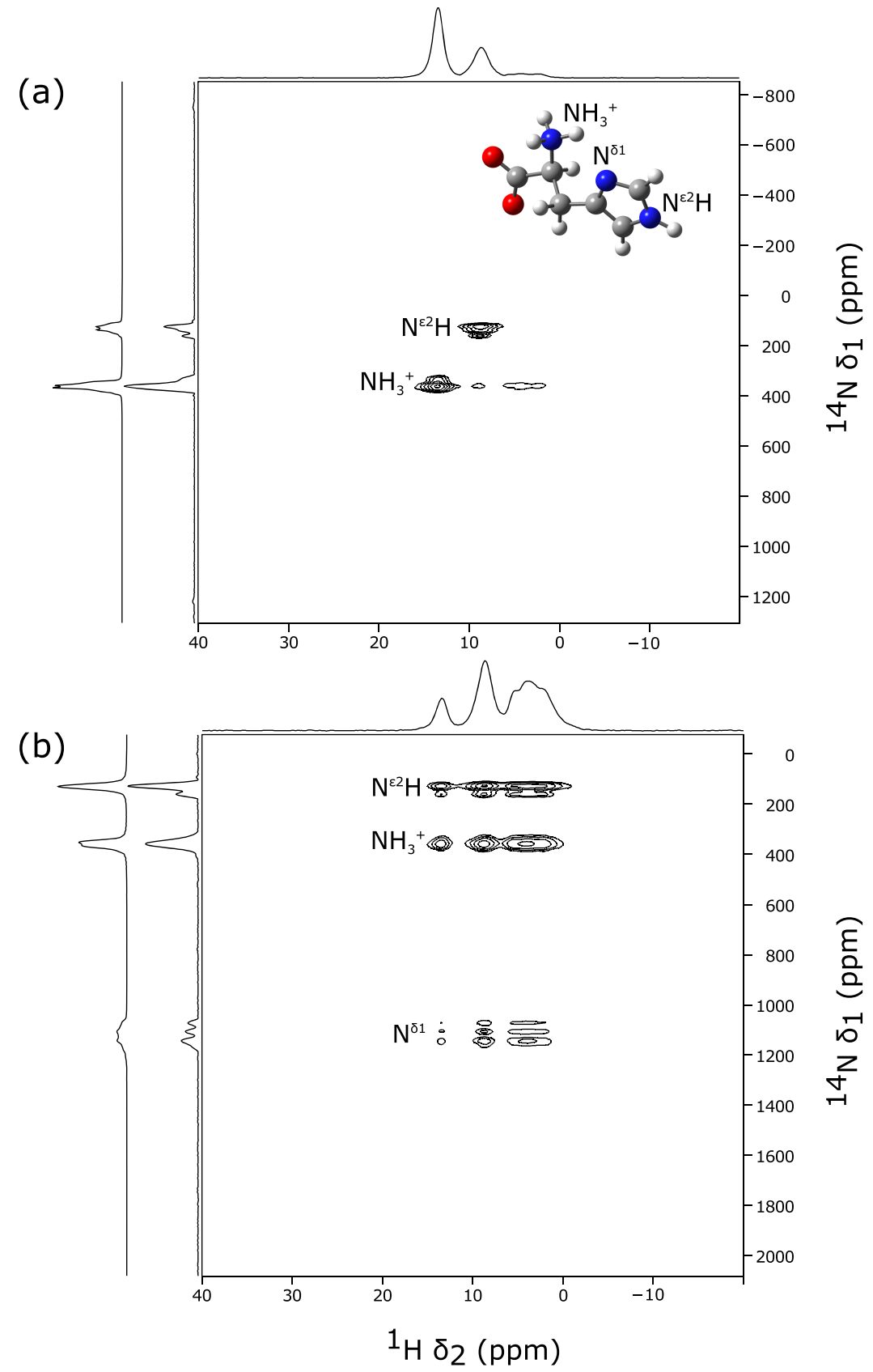

FIG. 2. (a) Experimental $2 \mathrm{D}{ }^{1} \mathrm{H}-{ }^{14} \mathrm{~N}$ single-quantum correlation spectrum of L-histidine at $\mathrm{B}_{0}=9.4 \mathrm{~T}(400.1$ $\mathrm{MHz}$ for ${ }^{1} \mathrm{H}, 28.9 \mathrm{MHz}$ for ${ }^{14} \mathrm{~N}$ ) recorded with a spinning frequency of $v_{\mathrm{R}}=62.5 \mathrm{kHz}$ (rotor period $\tau_{\mathrm{R}}=16 \mu \mathrm{s}$ ) and with a contact time of $\tau_{\mathrm{CP}}=15 \tau_{\mathrm{R}}=240 \mu \mathrm{s}$. The most intense one-bond correlations correspond to the $\mathrm{NH}_{3}{ }^{+}$ and $\mathrm{N}^{\varepsilon 2} \mathrm{H}$ sites. Weaker longer-range correlations corresponding to more distant protons can also be identified. (b) Spectrum recorded with a longer contact time of $\tau_{\mathrm{CP}}$ $=400 \tau_{\mathrm{R}}=6.4 \mathrm{~ms}$. The unprotonated nitrogen $\mathrm{N}^{\delta 1}$ of the imidazole moiety can now be identified. Numerical simulations of the projections onto the $\omega_{1}$ axes are shown on the left side for both cases. A recovery delay of $1 \mathrm{~s}$ was used and 64 transients were averaged in both cases. The number of $t_{1}$ increments was 200 for (a) and 160 for (b).
$\tau_{C P}=160 \mu \mathrm{s}=10 \tau_{R}$, which is most effective for one-bond transfers. Signals of both $\mathrm{NH}_{3}{ }^{+}$and amide $\mathrm{NH}$ fragments, which are expected to have very different $C_{Q}$ constants of ca. 1 and $3 \mathrm{MHz}$, respectively, can be clearly identified. This demonstrates that our method is efficient over a wide range of $C_{Q}$ values and is therefore suitable for detecting a wide variety of nitrogen-14 sites. Numerical simulations of the $\omega_{1}$ projections are shown on the left side along with the experimental ones, with relevant parameters given in the supplementary material.

In order to evaluate the sensitivity of our ${ }^{1} \mathrm{H} \rightarrow{ }^{14} \mathrm{~N} \rightarrow{ }^{1} \mathrm{H}$ DCP method, a ${ }^{1} \mathrm{H} \rightarrow{ }^{13} \mathrm{C} \rightarrow{ }^{1} \mathrm{H}$ DCP spectrum was acquired for comparison on a sample of ${ }^{13} \mathrm{C}$ - and ${ }^{15} \mathrm{~N}$-enriched $\mathrm{L}$ histidine. The grey contours in Fig. 3(b) show signals of all four ${ }^{1} \mathrm{H}_{\mathrm{n}}{ }^{13} \mathrm{C}$ groups. A contact time $\tau_{C P}=96 \mu \mathrm{s}=6 \tau_{R}$ was chosen to maximize one-bond transfers. A ${ }^{1} \mathrm{H} \rightarrow{ }^{14} \mathrm{~N} \rightarrow{ }^{1} \mathrm{H}$ DCP spectrum of natural-abundance L-histidine was acquired at the same spinning rate $v_{R}=62.5 \mathrm{kHz}$, with the same number of 32 scans and 64 increments in $t_{1}$ (so that $t_{1}{ }^{\text {max }}=64 \tau_{R}$ $\approx 1 \mathrm{~ms}$ ), the same recovery delay of $1 \mathrm{~s}$ and identical signal processing. The ${ }^{14} \mathrm{~N}$ spectrum is shown with blue contours overlaid on top of the grey ${ }^{13} \mathrm{C}$ spectrum. The corresponding projections for the two $2 \mathrm{D}$ spectra are shown along the sides using the same color-coding. No ${ }^{1} \mathrm{H}$ decoupling was applied in the evolution interval $t_{1}$ in either spectra. The digital resolution in $\omega_{1}$ is determined by $\left(t_{1}{ }^{\max }\right)^{-1}=\left(64 \tau_{R}\right)^{-1} \approx 1 \mathrm{kHz}$, which does not allow one to resolve any inhomogeneous features in the ${ }^{14} \mathrm{~N}$ line shapes. The more pronounced wiggles observed in the indirect $\omega_{1}$ dimension of the ${ }^{13} \mathrm{C}$ spectrum are due to digital truncation, indicating longer lifetimes of ${ }^{13} \mathrm{C}$ coherences compared with those of ${ }^{14} \mathrm{~N}$, i.e., $\mathrm{T}_{2}{ }^{\mathrm{SQC}}\left({ }^{13} \mathrm{C}\right)>\mathrm{T}_{2}{ }^{\mathrm{SQC}}\left({ }^{14} \mathrm{~N}\right)$. We observe a gain in intensity of ca. 3.5 when going from ${ }^{14} \mathrm{~N}$ to ${ }^{13} \mathrm{C}$ nuclei, close to the ratio $\gamma\left({ }^{13} \mathrm{C}\right) / \gamma\left({ }^{14} \mathrm{~N}\right)=3.48$. This shows that our technique allows us to obtain ${ }^{14} \mathrm{~N}$ spectra with 

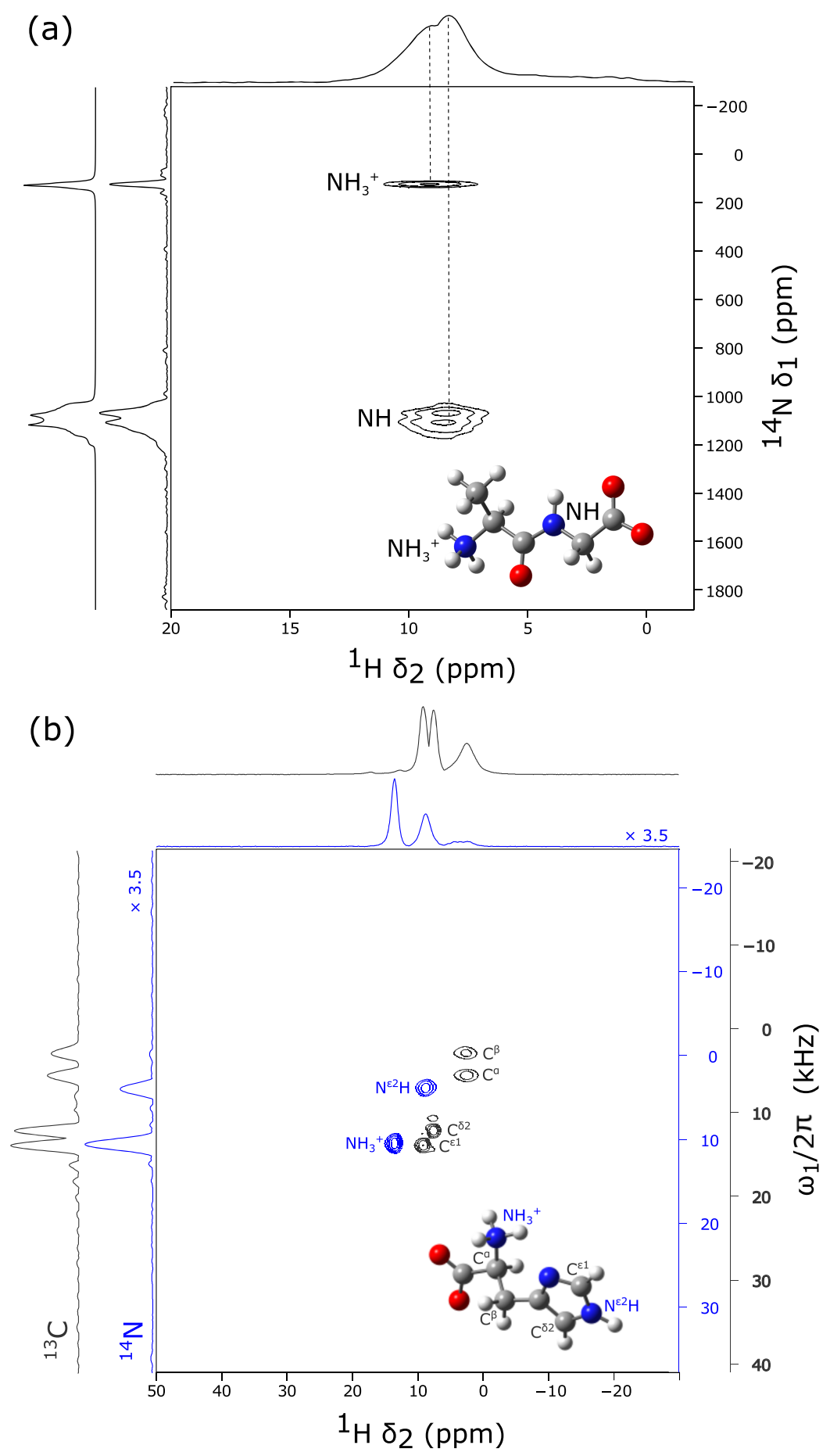

FIG. 3. (a) Experimental ${ }^{1} \mathrm{H}-{ }^{14} \mathrm{~N}$ single-quantum correlation spectrum of the dipeptide Ala-Gly at $B_{0}=9.4$ $\mathrm{T}$ recorded with a spinning frequency of $v_{\mathrm{R}}=62.5 \mathrm{kHz}$ $\left(\tau_{R}=16 \mu \mathrm{s}\right)$ and with a contact time of $\tau_{C P}=10 \tau_{R}=160$ $\mu \mathrm{s}$ and 64 transients for each of $160 t_{1}$ increments. Both one-bond correlations of the narrow $\mathrm{NH}_{3}{ }^{+}$and the broad NH sites can be identified. Numerical fits of the $\omega_{1}$ projections are shown on the left side. (b) Overlay of a ${ }^{1} \mathrm{H}-{ }^{14} \mathrm{~N}$ correlation spectrum of natural abundance L-histidine (blue: $\tau_{C P}=15 \tau_{R}=240 \mu \mathrm{s}$ ) and a ${ }^{1} \mathrm{H}-{ }^{13} \mathrm{C}$ correlation spectrum of ${ }^{13} \mathrm{C}$-enriched L-histidine (gray: $\left.\tau_{C P}=6 \tau_{R}=96 \mu \mathrm{s}\right)$. The ${ }^{1} \mathrm{H}-{ }^{14} \mathrm{~N}$ and ${ }^{1} \mathrm{H}-{ }^{13} \mathrm{C}$ spectra in (b) were recorded with exactly the same parameters for acquisition and processing: 32 transients were averaged and $64 t_{1}$ increments were recorded in both cases, in a total experimental time of ca. $34 \mathrm{~min}$. The protonated nitrogen-14 and carbon- 13 sites lead to signals with similar intensities.

an efficiency that is comparable to that of ${ }^{13} \mathrm{C}$ in ${ }^{13} \mathrm{C}$-enriched samples. Of course, larger rotor sizes of, say $4 \mathrm{~mm}$, spun at lower spinning rates of, say, $10 \mathrm{kHz}$, would surely grant higher sensitivity for $1 \mathrm{D}^{13} \mathrm{C} \mathrm{CP}$ spectra.

In order to investigate the efficiency of the ${ }^{1} \mathrm{H} \rightarrow{ }^{14} \mathrm{~N} \rightarrow{ }^{1} \mathrm{H}$ transfer by double cross polarization, experiments were conducted on polycrystalline powder samples of glycine and L-histidine. One can monitor the $\mathrm{CP}$ process as a function of the duration $\tau_{\mathrm{CP}}$ of the cross-polarization contact pulses and the $r f$-field strengths, in a manner that is very similar to conventional ${ }^{1} \mathrm{H}-{ }^{13} \mathrm{C}$ one-way cross polarization. Figure 4(a) shows proton signals observed with the DCP approach in glycine, with $\tau_{\mathrm{CP}}=320 \mu \mathrm{s}=n \tau_{R}$ with $n=20$, at a spinning rate of $v_{R}=62.5 \mathrm{kHz}\left(\tau_{R}=16 \mu \mathrm{s}\right)$ in steps of $\Delta \tau_{\mathrm{CP}}=\tau_{R}$. The black dots show the build-up of the $\mathrm{NH}_{3}{ }^{+}$proton signals, whereas empty circles show the $\mathrm{CH}_{2}$ proton signals. The former profile is characterized by a shorter build-up time due to the short one-bond distances involved in the ${ }^{1} \mathrm{H} \rightarrow{ }^{14} \mathrm{~N} \rightarrow{ }^{1} \mathrm{H}$ transfers (ca. $1.1 \AA$ ). By contrast, the latter profile shows a slower build-up since the protons involved in the $\mathrm{CP}$ process are found at longer distances from the ${ }^{14} \mathrm{~N}$ nucleus, i.e., ca. $2.1 \AA$ if we assume that the transfers occur within the same molecule. Interestingly, an inversion of the sign of the signals is observed for both pathways around $\tau_{\mathrm{CP}}$ $=5 \tau_{R}$. Figure 4(b) shows build up curves similar to those in Fig. 4(a) over a longer time scale $\tau_{\mathrm{CP}}=250 \tau_{R}=4 \mathrm{~ms}$ in 
(a)

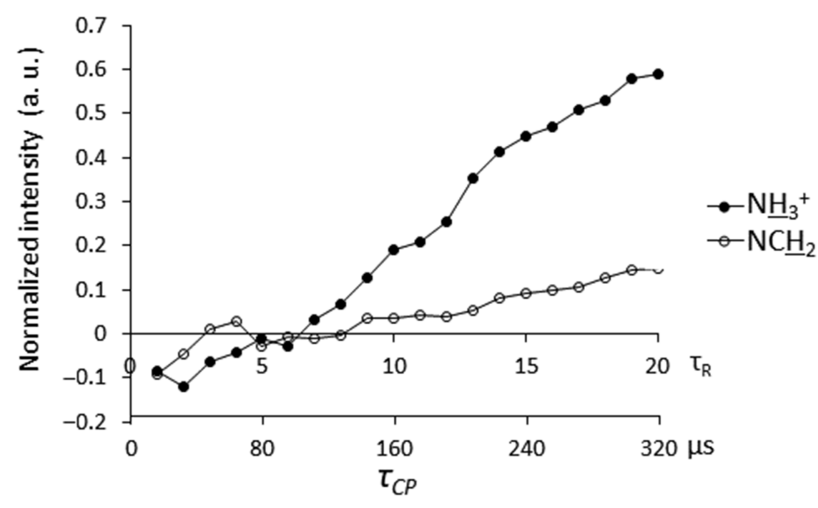

(c)

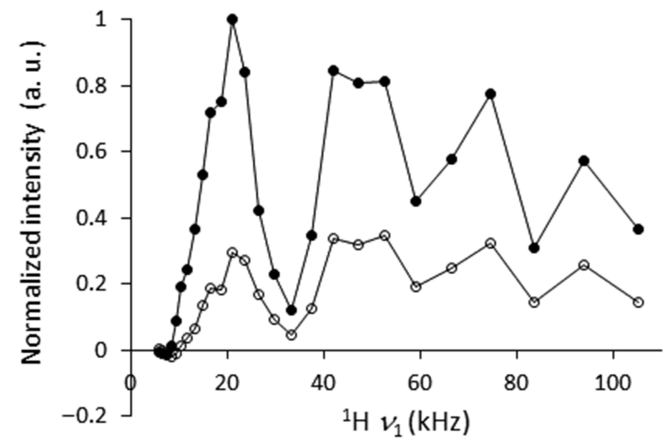

(b)

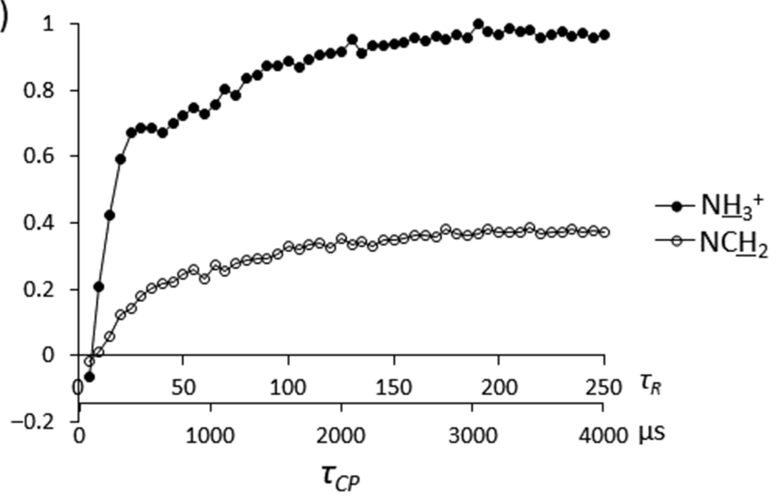

(d)

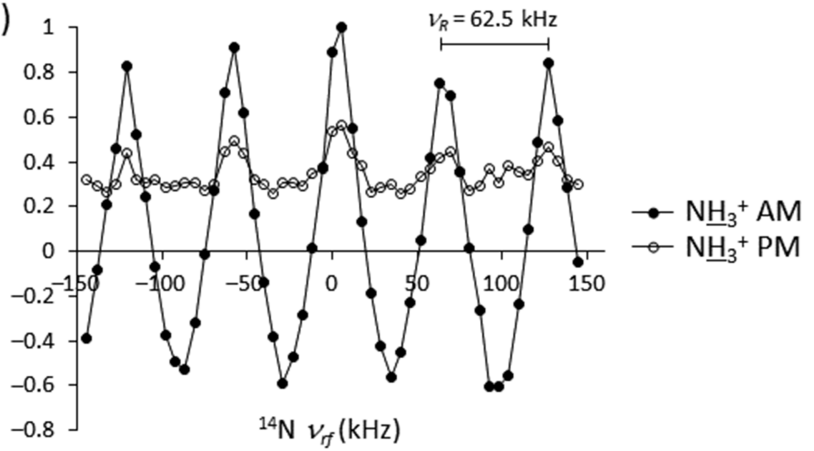

FIG. 4. (a) Experimental signal amplitudes of distinguishable protons of glycine $\left(\mathrm{NH}_{3}{ }^{+} \mathrm{CH}_{2} \mathrm{CO}_{2}{ }^{-}\right)$observed after a double ${ }^{1} \mathrm{H} \rightarrow{ }^{14} \mathrm{~N} \rightarrow{ }^{1} \mathrm{H}$ transfer as a function of the length of the contact pulses $\tau_{C P}=n \tau_{R}$, equal to an integer number $n$ of rotor periods $\tau_{R}=16 \mu \mathrm{s}$, for $1 \leq n \leq 20$ incremented in steps of 1 . Signals detected on the $\mathrm{NH}_{3}{ }^{+}$protons due to one-bond transfers are indicated by black dots, whereas signals detected on the $\mathrm{NC}^{\alpha} \mathrm{H}_{2}$ protons that arise from long-range transfers are indicated by empty circles. (b) Similar signals for $5 \leq n \leq 250$, incremented in steps of 5. (c) Similar signals recorded as a function of the $r f$ field strength $v_{1}\left({ }^{1} \mathrm{H}\right)$ applied to the proton channel during a contact time $\tau_{C P}=225 \tau_{R}$. (d) Similar signals recorded as a function of the nitrogen-14 carrier frequency $v_{r f}\left({ }^{14} \mathrm{~N}\right)$ during cross polarization. Both amplitude-modulated (AM) and phase-modulated (PM) experiments are shown.

steps of $\Delta \tau_{\mathrm{CP}}=5 \tau_{R}$. Filled and empty circles have the same meaning as in Fig. 1(a). The build-up curve of the $\mathrm{NH}_{3}{ }^{+}$signal reaches a plateau near $25 \tau_{R}<\tau_{\mathrm{CP}}<30 \tau_{R}$. Subsequently, a second surge of signal intensity attributed to more remote protons starts to contribute. This second component is similar to the build-up curve of the $\mathrm{CH}_{2}$ protons represented by the empty circles.

The conditions for matching the $r f$-amplitudes $v_{1}\left({ }^{1} \mathrm{H}\right)$ and $v_{1}\left({ }^{14} \mathrm{~N}\right)$ for the best ${ }^{1} \mathrm{H} \rightarrow{ }^{14} \mathrm{~N} \rightarrow{ }^{1} \mathrm{H}$ transfer were also investigated. Figure 4(c) shows signal intensities in one-dimensional DCP experiments carried out on glycine by varying the proton $r f$-field strength $v_{1}\left({ }^{1} \mathrm{H}\right)$ while keeping $v_{1}\left({ }^{14} \mathrm{~N}\right)=83 \mathrm{kHz}$. The length of the two contact intervals was $\tau_{\mathrm{CP}}=225 \tau_{R}=3.6 \mathrm{~ms}$. Filled and empty circles refer to signals detected on the $\mathrm{NH}_{3}{ }^{+}$ and $\mathrm{CH}_{2}$ protons, respectively, after transfer via ${ }^{14} \mathrm{~N}$, selected by phase cycling. The $r f$-matching profiles are very similar, with optimal transfer conditions appearing at intervals of ca. $23 \mathrm{kHz}$.

The robustness of DCP experiments with respect to the position of the ${ }^{14} \mathrm{~N}$ carrier frequency $v_{r f}\left({ }^{14} \mathrm{~N}\right)$, which was varied within the manifold of spinning sidebands that is determined by first-order quadrupolar couplings, was also investigated on the same glycine sample. Figure 4(d) shows signal intensities for DCP experiments performed by varying the carrier $v_{r f}\left({ }^{14} \mathrm{~N}\right)$ over a range of $289 \mathrm{kHz}$ (ca. $10000 \mathrm{ppm}$ for a ${ }^{14} \mathrm{~N}$ Larmor frequency of $28.9 \mathrm{MHz}$ at $\mathrm{B}_{0}=9.4 \mathrm{~T}$.) This range of $289 \mathrm{kHz}$ amounts to ca. $4.6 v_{R}$, i.e., 4.6 times the spectral width in the indirect dimension, which is identical to the spinning frequency $v_{R}=62.5 \mathrm{kHz}$ since the experiments are rotor-synchronized. Only the $\mathrm{NH}_{3}{ }^{+}$signals are shown for simplicity, the profiles of the $\mathrm{CH}_{2}$ protons being almost identical. Filled circles show modulations of the signal intensity with a periodicity that matches the spinning frequency $v_{R}=62.5$ $\mathrm{kHz}$, passing from positive to negative values. As remarked by O'Dell et al., ${ }^{48}$ this latter feature is related to the amplitude modulation (AM) of the signal if the evolution interval chosen in the one-dimensional DCP experiments is fixed (i.e., $t_{1}=\tau_{R}$ in the present case), as determined by the selection of both $p\left({ }^{14} \mathrm{~N}\right)= \pm 1$ coherence orders. In such $1 \mathrm{D}$ experiments, the signal may be written as $\mathrm{S}_{\mathrm{AM}}\left(t_{1}, t_{2}\right)=(1 / 2) \cos \left(\Omega_{\mathrm{S}} t_{1}\right)$ $\exp \left(i \Omega_{\mathrm{I}} t_{2}\right)$, where the fixed evolution interval is $t_{1}=\tau_{R}$. Since the offset in the $\omega_{1}$ dimension is $\Omega_{\mathrm{S}}=\omega_{0, \mathrm{~S}}+2 \pi v_{r f}\left({ }^{14} \mathrm{~N}\right)$, stepping the carrier frequency $v_{r f}\left({ }^{14} \mathrm{~N}\right)$ leads to the observed amplitude modulation. If one utilizes a phase-modulated (PM) version of the experiment by selecting only $p\left({ }^{14} \mathrm{~N}\right)=-1$ coherences in $t_{1}$, one obtains a nearly flat response, as shown by the empty circles in Fig. 4(d). In this case, one has $S_{\mathrm{PM}}\left(t_{1}\right.$, $\left.t_{2}\right)=(1 / 4) \exp \left(i \Omega_{\mathrm{S}} t_{1}\right) \exp \left(i \Omega_{\mathrm{I}} t_{2}\right)$, so that stepping the carrier frequency $v_{r f}\left({ }^{14} \mathrm{~N}\right)$ introduces a phase modulation. This can be compensated by a simple first-order phase correction after Fourier transformation. The two-fold reduction in amplitude observed when switching from amplitude- to phase-modulated experiments is because in the latter case half of the signal is discarded by the phase cycle. A PM version of the experiment may be preferred for the optimization of experimental parameters in $1 \mathrm{D}$ experiments but is not suitable as is for $2 \mathrm{D}$ 
spectroscopy since it results in phase-twisted 2D line shapes. The nearly flat responses prove the robustness of the DCP transfer for all nitrogen-14 sites that occur in the detected window, provided the carrier frequency $v_{r f}\left({ }^{14} \mathrm{~N}\right)$ is positioned within the inhomogeneous line-width that is due to the firstorder quadrupolar interactions in the static sample or within the manifold of sidebands in a spinning sample. This requirement can be easily fulfilled since the first-order quadrupolar broadening typically spans several MHz. Analogous responses acquired for L-histidine are substantially identical. Numerical data of these plots are given in the supplementary material.

We compared the performance of our DCP approach with the conventional HMQC method. Both experiments were optimized on a sample of glycine and subsequently applied to samples of L-histidine and the dipeptide Ala-Gly without reoptimizing the parameters (see the supplementary material). These results indicate that our DCP approach is less demanding in terms of the adjustment of the parameters since it performed well on all samples considered without the need for samplespecific optimizations. Furthermore, when a sufficient number of $t_{1}$ increments are acquired, the resulting 2 nd-order features of the ${ }^{14} \mathrm{~N}$ line shapes are better resolved in the DCP experiment than those produced by HMQC, with FWHH of ca. 1.3 and $1.9 \mathrm{kHz}$, respectively, obtained on the glycine sample (see the supplementary material). As discussed in the Introduction, we ascribe this latter finding to homogeneous losses due to transverse relaxation $T_{2}\left({ }^{1} \mathrm{H}\right)$ and to proton-proton dipole-dipole interactions that affect product states such as $T^{I}{ }_{1 \pm 1} T^{S}{ }_{1 \pm 1}$ and $T^{I}{ }_{1 \pm 1} T^{S}{ }_{2 \pm 1}$. Obviously, a refocusing pulse applied in the center of the $t_{1}$ evolution interval fails to cancel the effects of dipole-dipole proton-proton interactions, which may be regarded as the Achilles' heel of all HMQC methods. These interactions evidently cannot contribute to the decay of single-spin states such as $T^{S}{ }_{1 \pm 1}$.

We have attempted to tackle non-crystalline systems such as a lyophilized sample of lysozyme with disappointing results. This suggests that the distribution of molecular conformations and environments in amorphous solids-or a high number of crystallographically distinct sites in ordered materialsmay result in excessive broadening of ${ }^{14} \mathrm{~N}$ line shapes. In this respect, it is important to consider (i) the isotropic shifts due to second-order quadrupolar couplings that contribute to the spread of resonance frequencies if there is a range of $C_{Q}$ values and (ii) the fact that one must necessarily observe transitions $m_{I}= \pm 1 \leftrightarrow m_{I}=0$ that are broadened by the firstorder quadrupolar interaction, so that the width in the rotorsynchronized indirect dimension is limited by the spinning rate and may result in extensive folding and overlap. A computational investigation of these limitations is presented in the supplementary material, where we show how inhomogeneities in local environments result in a spread of resonances, which is relatively small for the isotropic chemical shift but much more severe for the quadrupolar interactions. These simulations show why amorphous samples may tend to give rise to featureless spectra because the ${ }^{14} \mathrm{~N}$ quadrupolar interactions may vary dramatically with the local environment.

All build-up plots in Fig. 4 show signal amplitudes observed in one-dimensional experiments resulting from the averaging of 32 transients. Considering the small amounts of ca. $3 \mathrm{mg}$ that can be packed in $1.3 \mathrm{~mm}$ rotors, the relatively small number of scans required for DCP experiments indicates that indirect detection of ${ }^{14} \mathrm{~N}$ via cross polarization at high spinning rates may be accomplished with an efficiency comparable to that of common direct one-dimensional ${ }^{13} \mathrm{C}$ detection with proton decoupling in ${ }^{13} \mathrm{C}$-enriched samples. Furthermore, the only parameters that need to be adjusted experimentally are exactly the same as one has to optimize for common ${ }^{13} \mathrm{C} \mathrm{CP}$ MAS spectra, i.e., the contact time $\tau_{C P}$ and $r f$-field strength $v_{1}$ of one of the two channels. These features indicate that our approach grants both maximal sensitivity and ease of use. Furthermore, the pulse sequence does not have to be symmetric about the center of the $t_{1}$ delay. Thus, a longer contact may be chosen for the first ${ }^{1} \mathrm{H} \rightarrow{ }^{14} \mathrm{~N}$ transfer to exploit the full proton bath as a source of polarization, and a shorter contact may be used after the delay to favor one-bond transfers. By inspection of the build-up profiles of Figs. 4(a) and 4(b) and Figs. 1(a) and 1(b) of the supplementary material, one may conclude that different contact lengths for the first and second CP steps may result in improvements or deteriorations in terms of signal intensity, which are site- as well as samplespecific. Rotor-synchronization of the length contact pulses is not required and their deliberate desynchronization has little effect on the signal intensity.

\section{CONCLUSIONS}

A simple double cross polarization (DCP) experiment provides an efficient approach for the indirect detection of nitrogen-14 NMR spectra under fast magic-angle spinning conditions on polycrystalline samples such as glycine, L-histidine, and the dipeptide Ala-Gly. The resulting twodimensional correlation spectra can be tuned to unveil either short-range one-bond or long-range correlations. In the latter case, unprotonated ${ }^{14} \mathrm{~N}$ sites can be detected. The method is shown experimentally to be effective over a range of quadrupolar coupling constants of at least 1-4 MHz. Amorphous samples tend to give rise to very broad ${ }^{14} \mathrm{~N}$ spectra because the quadrupolar interactions are very sensitive to the lattice environment. The method is straightforward to implement since it only requires a few simple optimizations, much like standard $\mathrm{CP}$ methods that are used routinely for ${ }^{13} \mathrm{C}$ and other nuclei with $S=1 / 2$. The efficiency of the polarization transfer has been investigated as a function of the length of the contact pulses, the $r f$-field strength, and $r f$-carrier frequency. The efficiency of the transfer of polarization and the sensitivity in our ${ }^{1} \mathrm{H}-{ }^{14} \mathrm{~N}$ experiments is comparable to similar ${ }^{1} \mathrm{H}-{ }^{13} \mathrm{C}$ experiments of samples that are isotopically enriched in carbon-13.

\section{EXPERIMENTAL DETAILS}

All DCP MAS spectra were recorded at the Ecole Normale Supérieure de Paris, on a wide-bore Bruker 400 spectrometer (9.4 T) with an Avance-III console, using $1.3 \mathrm{~mm} \mathrm{ZrO}_{2}$ rotors spinning at $v_{R}=62.5 \mathrm{kHz}$ in a triple-resonance MAS probe in a two-channel configuration. Typical amounts of samples packed in the rotors were ca. $3 \mathrm{mg}$ (for L-histidine). The $r f$ field amplitude for proton excitation pulses was $v_{1}=178 \mathrm{kHz}$, corresponding to $90^{\circ}$ flip-angle pulse length of $\tau_{p}=1.4 \mu \mathrm{s}$. 
The $r$-field strengths for ${ }^{1} \mathrm{H} \rightarrow{ }^{14} \mathrm{~N} \rightarrow{ }^{1} \mathrm{H}$ cross polarization steps were $v_{1}\left({ }^{1} \mathrm{H}\right)=47$ and $v_{1}\left({ }^{14} \mathrm{~N}\right)=83 \mathrm{kHz}$, whereas those for ${ }^{1} \mathrm{H} \rightarrow{ }^{13} \mathrm{C} \rightarrow{ }^{1} \mathrm{H}$ were $v_{1}\left({ }^{1} \mathrm{H}\right)=66$ and $v_{1}\left({ }^{13} \mathrm{C}\right)=50 \mathrm{kHz}$. A recycling delay of $1 \mathrm{~s}$ was used in all cases. Phase cycling for the DCP experiment: $\phi_{1}=4\{y\}, 4\{-y\}, 4\{y\}, 4\{-y\}$, $\phi_{2}=8\{x\}, 8\{-x\}, \phi_{3}=4\{x,-x\}, 4\{-x, x\}, \phi_{4}=2\{x\}, 2\{-x\}$, $\phi_{5}=\mathrm{x}, \phi_{\text {rec }}=\mathrm{x},-\mathrm{x},-\mathrm{x}, \mathrm{x},-\mathrm{x}, \mathrm{x}, \mathrm{x},-\mathrm{x},-\mathrm{x}, \mathrm{x}, \mathrm{x},-\mathrm{x}, \mathrm{x},-\mathrm{x},-\mathrm{x}, \mathrm{x}$ (see the supplementary material). The amplitude of the pulses was kept constant during all $\mathrm{CP}$ steps. No ${ }^{1} \mathrm{H}$ decoupling was applied during the $\mathrm{t}_{1}$ interval (see the supplementary material). The chemical shifts were referenced with respect to adamantane and $\mathrm{NH}_{4} \mathrm{Cl}$ whereas the magic angle was adjusted using $\mathrm{KBr}$.

\section{SUPPLEMENTARY MATERIAL}

See supplementary material for details and discussion about profiles analogous to those of Fig. 4 recorded on a sample of L-histidine, comparison between linewidths produced by the DCP and HMQC approaches, the effect of distribution of environments in terms of chemical shift and quadrupolar interaction, the effect of proton decoupling during $\mathrm{t}_{1}$ evolution, spurious signal eliminated by the adopted phase cycling, and considerations on the experimental times.

\section{ACKNOWLEDGMENTS}

The authors are indebted to Dr. Philippe Pelupessy and Dr. Nicolas Bilirakis for helpful discussions and assistance with experiments. Professor Paul Vasos and the University René Descartes (Paris-5, UMR 8601) contributed to funding. This work was supported by the CNRS, the European Research Council (ERC, contract "dilute para-water," Grant Agreement No. 339754), and the French "Equipements d'Excellence" Paris-en-Resonance, Contract No. ANR-10-EQPX-09.

${ }^{1}$ M. J. Duer, Solid-State NMR Spectroscopy: Principles and Applications (Blackwell Science, UK, 2002).

${ }^{2}$ S. R. Hartmann and E. L. Hahn, "Nuclear double resonance in the rotating frame," Phys. Rev. 128, 2042-2053 (1962).

${ }^{3}$ A. Pines, M. G. Gibby, and J. S. Waugh, "Proton-enhanced NMR of dilute spins in solids," J. Chem. Phys. 59, 569-590 (1973).

${ }^{4}$ L. Frydman and J. S. Harwood, "Isotropic spectra of half-integer quadrupolar spins from bidimensional magic-angle spinning NMR," J. Am. Chem. Soc. 117, 5367-5368 (1995).

${ }^{5} \mathrm{Z}$. Gan, "Isotropic NMR spectra of half-integer quadrupolar nuclei using satellite transitions and magic-angle spinning," J. Am. Chem. Soc. 122, 3242-3243 (2000).

${ }^{6}$ M. Pruski, D. P. Lang, C. Fernandez, and J. P. Amoureux, "Multiplequantum magic-angle spinning NMR with cross-polarization: Spectral editing of high-resolution spectra of quadrupolar nuclei," Solid State Nucl. Magn. Reson. 7, 327-331 (1997).

${ }^{7}$ C. Fernandez, L. Delevoye, and J. P. Amoureux, “ ${ }^{27} \mathrm{Al}\left\{{ }^{1} \mathrm{H}\right\}$ cross polarization triple-quantum magic angle spinning NMR, ’ J. Am. Chem. Soc. 119, 6858-6862 (1997).

${ }^{8}$ S. H. Wang, S. M. De Paul, and L. M. Bull, "High-resolution heteronuclear correlation between quadrupolar and spin-1/2 nuclei using multiple-quantum magic-angle spinning," J. Magn. Reson. 125, 364-368 (1997).

${ }^{9}$ S. E. Ashbrook, S. P. Brown, and S. Wimperis, "Multiple-quantum crosspolarization in MAS NMR of quadrupolar nuclei," Chem. Phys. Lett. 288, 509-517 (1998).

${ }^{10}$ S. E. Ashbrook and S. Wimperis, "Single- and multiple-quantum crosspolarization in NMR of quadrupolar nuclei in static samples," Mol. Phys. 98, 1-26 (2000).
${ }^{11}$ L. Muller, "Proton-deuterium polarization transfer in magic angle spinning polycrystalline solids in the rotating frame," Chem. Phys. 61, 235-248 (1981).

${ }^{12} \mathrm{~K}$. Gopalakrishnan and G. Bodenhausen, "Cross polarization from spins $\mathrm{I}=1 / 2$ to spins $\mathrm{S}=1$ in nuclear magnetic resonance with magic angle spinning," J. Chem. Phys. 124, 194311 (2006).

${ }^{13}$ K. Basse, S. K. Jain, O. Bakharev, and N. C. Nielsen, "Efficient polarization transfer between spin-1/2 and ${ }^{14} \mathrm{~N}$ nuclei in solid-state MAS NMR spectroscopy," J. Magn. Reson. 244, 85-89 (2014).

${ }^{14}$ E. A. Hill and J. P. Yesinowski, "Wide-line 14N NMR in solids and reorientation-induced redistribution of isochromats," J. Am. Chem. Soc. 118, 6798-6799 (1996).

${ }^{15}$ L. A. O'Dell and R. W. Schurko, "Fast and simple acquisition of solid-state 14N NMR spectra with signal enhancement via population transfer," J. Am. Chem. Soc. 131, 6658-6659 (2009).

${ }^{16}$ L. A. O'Dell and R. W. Schurko, "Static solid-state 14N NMR and computational studies of nitrogen EFG tensors in some crystalline amino acids," Phys. Chem. Chem. Phys. 11, 7069-7077 (2009).

${ }^{17}$ L. A. O’Dell, R. W. Schurko, K. J. Harris, J. Autschbach, and C. I. Ratcliffe, "Interaction tensors and local dynamics in common structural motifs of nitrogen: A solid-state 14N NMR and DFT study," J. Am. Chem. Soc. 133, 527-546 (2011).

${ }^{18}$ H. J. Jakobsen, H. Bildsøe, J. Skibsted, and T. Giavani, "14N MAS NMR spectroscopy: The nitrate ion," J. Am. Chem. Soc. 123, 5098-5099 (2001).

${ }^{19}$ H. J. Jakobsen, H. Bildsøe, Z. Gan, and W. W. Brey, "Experimental aspects in acquisition of wide bandwidth solid-state MAS NMR spectra of low- $\gamma$ nuclei with different opportunities on two commercial NMR spectrometers," J. Magn. Reson. 211, 195-206 (2011).

${ }^{20}$ M. Bloom and M. A. LeGros, "Direct detection of two-quantum coherence," Can. J. Phys. 64, 1522-1528 (1986).

${ }^{21}$ R. Tycko and S. J. Opella, "High-resolution $14 \mathrm{~N}$ overtone spectroscopy: An approach to natural abundance nitrogen NMR of oriented and polycrystalline systems," J. Am. Chem. Soc. 108, 3531-3532 (1986).

${ }^{22}$ R. Tycko, P. L. Stewart, and S. J. Opella, "Peptide plane orientations determined by fundamental and overtone 14N NMR," J. Am. Chem. Soc. 108, 5419-5425 (1986).

${ }^{23}$ R. Tycko and S. J. Opella, “Overtone NMR spectroscopy,” J. Chem. Phys. 86, 1761-1774 (1987).

${ }^{24}$ L. A. O'Dell and C. I. Ratcliffe, "14N magic angle spinning overtone NMR spectra," Chem. Phys. Lett. 514, 168-173 (2011).

${ }^{25}$ L. A. O'Dell and A. Brinkmann, "14N overtone NMR spectra under magic angle spinning: Experiments and numerically exact simulations," J. Chem. Phys. 138, 064201 (2013).

${ }^{26}$ Y. Nishiyama, M. Malon, Z. Gan, Y. Endo, and T. Nemoto, "Protonnitrogen-14 overtone two-dimensional correlation NMR spectroscopy of solid-sample at very fast magic angle sample spinning," J. Magn. Reson. 230, 160-164 (2013).

${ }^{27}$ A. J. Rossini, L. Emsley, and L. A. O’Dell, "Dynamic nuclear polarization enhanced 14N overtone MAS NMR spectroscopy," Phys. Chem. Chem. Phys. 16, 12890-12899 (2014).

${ }^{28}$ I. M. Haies, J. A. Jarvis, H. Bentley, I. Heinmaa, I. Kuprov, P. T. F. Williamson, and M. Carravetta, "14N overtone NMR under MAS: Signal enhancement using symmetry-based sequences and novel simulation strategies," Phys. Chem. Chem. Phys. 17, 6577-6587 (2015).

${ }^{29} \mathrm{Z}$. Gan, "Measuring amide nitrogen quadrupolar coupling by high resolution 14N/13C NMR correlation under magic-angle spinning," J. Am. Chem. Soc. 128, 6040-6041 (2006).

${ }^{30}$ S. Cavadini, A. Lupulescu, S. Antonijevic, and G. Bodenhausen, "14N NMR spectroscopy using residual dipolar splittings in solids," J. Am. Chem. Soc. 128, 7706-7707 (2006).

${ }^{31}$ S. Cavadini, S. Antonijevic, A. Lupulescu, and G. Bodenhausen, "Indirect detection of $14 \mathrm{~N}$ in solids via protons by nuclear magnetic resonance spectroscopy,” J. Magn. Reson. 182, 168-172 (2006).

${ }^{32}$ S. Cavadini, S. Antonijevic, A. Lupulescu, and G. Bodenhausen, "Indirect detection of $14 \mathrm{~N}$ in solid-state NMR spectroscopy," Chem. Phys. Chem. 8, 1363-1374 (2007).

${ }^{33} \mathrm{Z}$. Gan, "13C/14N hetero-nuclear multiple-quantum correlation with rotary resonance and REDOR dipolar recoupling," J. Magn. Reson. 184, 39-43 (2006).

${ }^{34}$ Z. Gan, J. P. Amoureux, and J. Trébosc, "Proton-detected 14N MAS NMR using homo-nuclear decoupled rotary resonance," Chem. Phys. Lett. 435, 163-169 (2007). 
35 J. A. Jarvis, I. M. Haies, P. T. F. Williamson, and M. Carravetta, "An efficient NMR method for the characterisation of $14 \mathrm{~N}$ sites through indirect $13 \mathrm{C}$ detection," Phys. Chem. Chem. Phys. 15, 7613-7620 (2013).

${ }^{36}$ Y. Nishiyama, Y. Endo, T. Nemoto, H. Utsumi, K. Yamauchi, K. Hioka, and T. Asakura, "Very fast magic angle spinning ${ }^{1} \mathrm{H}-{ }^{14} \mathrm{~N} 2 \mathrm{D}$ solid-state NMR: Sub-micro-liter sample data collection in a few minutes," J. Magn. Reson. 208, 44-48 (2011).

${ }^{37}$ S. Cavadini, A. Abraham, and G. Bodenhausen, "Proton-detected 14N NMR by recoupling of hetero-nuclear dipolar interactions using symmetry-based sequences," Chem. Phys. Lett. 445, 1-5 (2007).

${ }^{38}$ S. Cavadini, V. Vitzthum, S. Ulzega, A. Abraham, and G. Bodenhausen, "Line-narrowing in proton-detected nitrogen-14 NMR," J. Magn. Reson. 202, 57-63 (2010).

${ }^{39}$ S. Cavadini, A. Abraham, and G. Bodenhausen, "Coherence transfer between spy nuclei and $14 \mathrm{~N}$ in solids," J. Magn. Reson. 190, 160-164 (2008).

${ }^{40}$ M. Shen, J. Trébosc, L. A. O’Dell, O. Lafon, F. Pourpoint, B. Hu, Q. Chen, and J. P. Amoureux, "Comparison of various NMR methods for the indirect detection of nitrogen-14 nuclei via protons in solids," J. Magn. Reson. 258, 86-95 (2015).

${ }^{41}$ V. Vitzthum, M. A. Caporini, S. Ulzega, and G. Bodenhausen, "Broadband excitation and indirect detection of nitrogen-14 in rotating solids using delays alternating with nutation (DANTE)," J. Magn. Reson. 212, 234-239 (2011).

${ }^{42}$ V. Vitzthum, M. A. Caporini, S. Ulzega, J. Trébosc, O. Lafon, J. P. Amoureux, and G. Bodenhausen, "Uniform broadband excitation of crystallites in rotating solids using interleaved sequences of delays alternating with nutation," J. Magn. Reson. 223, 228-236 (2012).

${ }^{43}$ M. Shen, J. Trébosc, O. Lafon, Z. Gan, F. Pourpoint, B. Hu, Q. Chen, and J. P. Amoureux, "Solid-state NMR indirect detection of nuclei experiencing large anisotropic interactions using spinning sideband-selective pulses," Solid State Nucl. Magn. Reson. 72, 104-117 (2015).

${ }^{44}$ L. A. O'Dell, "Direct detection of $14 \mathrm{~N}$ in solid-state NMR spectroscopy," Prog. Nucl. Magn. Reson. Spectrosc. 59, 295-318 (2011).

${ }^{45} \mathrm{~S}$. Cavadini, "Indirect detection of nitrogen-14 in solid-state NMR spectroscopy,” Prog. Nucl. Magn. Reson. Spectrosc. 56, 46-77 (2010).

${ }^{46}$ P. Brunner, M. Reinhold, and R. R. Ernst, "Double quantum cross polarization. Heteronuclear excitation and detection of NMR double quantum transitions in solids," J. Chem. Phys. 73, 1086-1094 (1980).

${ }^{47}$ M. Reinhold, P. Brunner, and R. R. Ernst, "Double quantum cross polarization via dipolar order," J. Chem. Phys. 74, 184-188 (1981).

${ }^{48}$ L. A. O'Dell, R. He, and J. Pandohee, "Identifying H-N proximities in solid-state NMR using 14N overtone irradiation under fast MAS," CrystEngComm 15, 8657-8667 (2013). 